\title{
Inhaling the Nation: \\ The Cultural Translation and Symbolic Performance of the Cigar in Cuba
}

\author{
Stephen Cruikshank \\ University of Alberta, Edmonton
}

The following article uses a mixture of poetry and text to trace the cigar through various stages of Cuban history and highlights how the cigar has been translated and as a symbol useful to the construction of Cuban nationalism. In what ways does the cultural representation of the cigar throughout Cuban history create a performance of cultural values, identities, and heritage? As this paper reveals, such a question require us to translate the cigar smoke, to breathe in Cuban history, and to exhale the performance of metaphors. 